\title{
Viscoelasticity of the polydomain-monodomain transition in main-chain liquid crystal elastomers
}

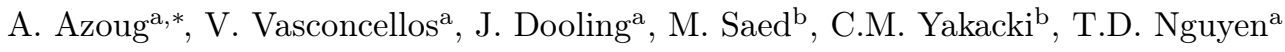 \\ ${ }^{a}$ Johns Hopkins University, Department of Mechanical Engineering, Baltimore, MD, USA. \\ ${ }^{b}$ University of Colorado Denver, Department of Mechanical Engineering, Denver, CO, USA.
}

\begin{abstract}
The goal of this study was to explore the rate-dependent behavior of the stretch-induced polydomainmonodomain (PM) transition of a liquid crystal elastomer (LCE). The main-chain LCE was synthesized and then cross-linked in the nematic polydomain state. The PM transition caused a soft-elastic behavior, which was measured using uniaxial tensile tests at multiple strain rates and temperatures. The main finding was that we were able to apply the temperature-dependent shift factor determined for the small strain behavior and in the frequency domain to create master curves for the large-strain response in the strain rate domain. The soft elasticity phenomenon was absent from the stress-strain curve at equilibrium. The results also suggest that the relaxation mechanisms of the network, and not of the mesogen orientation, dominate the rate-dependent behavior. Finally, we observed a relatively slow recovery behavior, suggesting the presence of an additional slow relaxation mechanism.
\end{abstract}

Keywords: liquid crystal elastomers, soft elasticity, polydomain, monodomain, viscoelasticity

\section{Introduction}

Liquid crystal elastomers (LCEs) are networks of cross-linked polymer chains containing rodlike molecules, called mesogens, in sufficient quantity to induce nematic liquid crystallinity under thermodynamically favorable conditions [1]. The mesogens are either incorporated into the polymer backbone or linked as a side group, leading to mainchain or side-chain LCEs, respectively $[2,3]$. The network provides solid-like behavior, which distinguishes LCEs from more conventional liquid crystals. LCEs typically reside in a rubbery state at room temperature, which allows mesogens to rotate and orient to accommodate an applied deformation or temperature transition. The coupling between the entropic elasticity of the network and the liquid crystal behavior of the mesogens gives rise to interesting thermomechanical behaviors including soft elasticity [4] and shape changes induced by the nematic-isotropic phase transition at $T=T_{n i}[3,5]$.

\footnotetext{
* Corresponding author

Email address: aurelie.azoug@polytechnique.edu (T.D. Nguyen)
}

Preprint submitted to Elsevier
LCEs can be synthesized into a polydomain or monodomain state, depending on whether or not the mesogens were aligned during network formation $[2,6,7]$. When no deformation is imposed during crosslinking, the resultant material presents a polydomain texture [2, 7]. Although polydomain LCEs are macroscopically isotropic, they possess a local orientation in each domain, which has a typical size of the order of a few microns [8]. Polydomain LCEs can be stretched to align the mesogens into a monodomain. This corresponds to the polydomain-monodomain (PM) transition, which can be observed by a change in optical transparency.

Most studies focused on characterizing the PM transition at equilibrium for side-chain LCEs, either using very low strain rates or applying small load increments separated by long equilibration time. The experimental findings showed that the state of the material, nematic or isotropic, during cross-linking has a large impact on the domain structure and the stress response during the PM transition. Higaki et al. showed the domain size is significantly smaller for LCEs crosslinked in the isotropic than nematic state [9]. At the PM transition, the stress

May 30, 2016 
response of polydomain LCEs with isotropic genesis typically contains 3 regions: 1) an initial elastic region, 2) a soft elasticity plateau that corresponds to the PM transition, and 3) a strain-hardening region [8]. For polydomain LCEs with nematic genesis, the PM transition does not result in a soft elasticity plateau and the stress response does not exhibit the 3 regions. The PM transition under equilibrium conditions gradually proceeds over a range of stress with no apparent plateau or soft elasticity [10-13]. However, away from equilibrium conditions, i.e. at lab-scale strain rates, the polydomain LCEs with nematic genesis exhibit the same qualitative stress response as those with isotropic genesis $[7,10,11,14-17]$.

In all studies measuring the influence of the temperature on the PM transition but one, the transition stress decreased with increasing temperature [7, 10, 14-17]. In some cases, the width of the transition also decreased with increasing temperature $[7,14,17]$. These observations agreed with the model proposed by Fridrikh and Terentjev [8] where the stress level and end strain of the PM transition depend on chain anisotropy, which decreases as the temperature approaches $T_{n i}$. In experimental approaches evaluating the influence of strain rate $[10,18]$, a plateau corresponding to the PM transition was measured on the stress-strain curve when the strain rate of the loading increased away from equilibrium conditions. In both studies [10, 18], the range of strain rates explored was limited to relatively low values, with a maximum at $0.03 \% / \mathrm{s}$ and $0.046 \% / \mathrm{s}$, respectively. In addition, the transition stress was either independent of strain rate $[1,13]$ or increased with the strain rate [10].

The relaxation mechanisms of side-chain polydomain nematic LCEs have been investigated using stress relaxation $[10,18]$ and strain recovery [19]. The stress relaxation and strain recovery curves are analyzed by fitting to a relaxation function to determine the distribution of relaxation times and infer the relaxation mechanisms. Three relaxation mechanisms were detected. Hotta and Terentjev [10] showed that side-chain polydomain LCEs exhibit long time relaxation dynamics, which they attributed to the relaxation behavior of the polymer network, while the relaxation stemming from mesogen orientation occured at short times, smaller than 1000s. Schonstein et al. [20] used dynamic light scattering of monodomain LCEs to measure the relaxation of the director orientation. Their analysis showed a broad distribution of relaxation times with a mean relaxation time of the order $0.01 \mathrm{~s}$ at $50^{\circ} \mathrm{C}$.

Comparing the recovery of swollen polydomain and monodomain LCEs, Urayama et al. [19] showed that the polydomain LCEs with nematic genesis exhibited faster relaxation times than those with isotropic genesis. Moreover, the relaxation times of the polydomain LCE were significantly slower than that of the monodomain LCE. They conclude that the slow strain recovery of polydomain LCEs reflects the slow recovery to the initial disordered polydomain texture. Similarly, Clarke and Terentjev [18] attributed the slow relaxation dynamics at long times to the cooperative motion of the domains to overcome the strain incompatibility caused by domain reorientation. To the best of our knowledge, few studies have examined the relaxation behavior of nematic main-chain polymers. The few studies of main chain networks have been performed on smectic LCEs, which can exhibit different relaxation and dissipative mechanisms than nematic LCEs [16, 21].

The goal of this study was to determine the contribution of the various relaxation mechanisms to the stretch-induced PM transition of main-chain LCEs with nematic genesis during loading, unloading, and recovery. We first demonstrated that the time-temperature superposition (TTS) shift factor determined for small strain in the frequency domain can be applied to the large strain stress response in the strain rate domain. We then measured the influence of strain rate and temperature, showing that the strain and stress levels at the onset and end of the PM transition obey the TTS principle. Finally, we discussed the relaxation mechanisms that may contribute to the non-equilibrium behavior at loading, unloading, and during strain recovery.

\section{Experimental}

\subsection{Materials}

The material studied is a main-chain polydomain LCE with nematic genesis formed by the Michael addition of di-thiol spacers, tetra-thiol crosslinkers, and di-acrylate mesogens [22, 23] (Fig. 1). The thiol monomers, pentaerythritol tetrakis (3-mercaptopropionate) (PETMP) and 2,2'-(ethylenedioxy) diethanethiol (EDDET), were purchased from Sigma Aldrich (St. Louis, MO, USA). Thiol solutions containing $13 \mathrm{~mol} \%$ PETMP functional groups were mixed (i.e. $13 \%$ of the overall thiol groups belonged to PETMP). 


\section{Di-acrylate mesogen (RM257)}<smiles>C=CC(=O)OCCCOc1ccc(OC(=O)c2ccc(OC(=O)c3ccc(OC(=O)c4ccc(OCCCOC(=O)C=C)cc4)cc3)cc2)cc1</smiles>

Di-thiol spacer (EDDET)<smiles>SCCOCCOCCS</smiles>

Tetra-thiol crosslinker (PETMP)

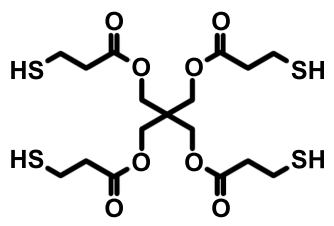

Figure 1: A di-acrylate mesogen, a di-thiol flexible spacer, and a tetra-thiol crosslinker

The di-acrylate mesogen, 1,4-Bis-[4(3-acryloyloxypropyloxy)benzoyloxy]-2-

methylbenzene (RM257), was purchased from Wilshire Technologies (Princeton, NJ, USA). The RM257 was dissolved in $31 \mathrm{wt} \%$ of toluene at $80^{\circ} \mathrm{C}$ for $5 \mathrm{~min}$. The RM257 was then added to the thiol solutions to create a non-stoichiometric acrylate:thiol functional group ratio of 1.15:1 (excess of $15 \%$ extra acrylate groups relative to thiol groups).

The catalyst dipropyl amine (DPA) and the photoinitiator (2-hydroxyethoxy)-2methylpropiophenone (HHMP) were purchased from Sigma Aldrich. The DPA was first mixed in toluene at a ratio of $1: 100.0 .23 \mathrm{~mol} \%$ DPA were added to the solution. For the photoinitiator, 0.5 wt $\%$ of the total monomer weight was added to the solution and mixed vigorously to ensure a homogenous mixture.

The solution was placed in a vacuum oven for a couple of minutes to remove any air bubbles, poured into dog-bone moulds, and allowed to cure in open air. The specimen was then dried for several days to evaporate the remaining solvent. The second-stage photopolymerization of the excess acrylate was performed using UV light at room temperature, which was below $T_{n i}$. No mechanical loading was applied during the second-stage cross-linking and the obtained specimens were in the polydomain state.

\subsection{Dynamic mechanical analysis}

The temperature-dependent storage modulus was measured using dynamic mechanical analysis (TA Instrument Q800). A specimen of size $7.0 \mathrm{~mm}$ $\times 3.0 \mathrm{~mm} \times 1.8 \mathrm{~mm}$ was cut and secured with film tension grips. The temperature was increased to $80^{\circ} \mathrm{C}$ and equilibrated for 15 minutes before subjecting the specimens to a frequency sweep between 0.15 and $40 \mathrm{~Hz}$ at $0.1 \%$ strain amplitude. The temperature was lowered to $-20^{\circ} \mathrm{C}$ in increments and the frequency sweep procedure repeated at each temperature increment.

\subsection{Tensile testing}

Tensile tests were performed in an MTS Insight 5 equipped with an MTS 500N force transducer, an environmental chamber, and a Eurotherm controller 2408. One dumbbell specimen of length 15.5 $\mathrm{mm}$ and section $2.8 \mathrm{~mm} \times 1.8 \mathrm{~mm}$ was used for all the uniaxial tensile tests.

The specimen was placed in tensile screw grips and uniaxially stretched from 0 to $100 \%$ engineering strain, which was sufficient to observe a complete PM transition. The specimen became fully transparent in the monodomain state by $100 \%$ strain. The material was then unloaded until zero force was recorded. Finally, the force was maintained at zero and the strain was measured to study the recovery response. The recovery time was defined as the time at which the engineering strain reached $2 \%$.

After each tensile test, the specimen was placed in an oven at $120^{\circ} \mathrm{C}$ for $15 \mathrm{~min}$ and subsequently left at room temperature for $15 \mathrm{~min}$. This thermal treatment between each test erased the deformation history of the specimen.

At room temperature, the specimen was uniaxially stretched from 0 to $100 \%$ engineering strain at multiple engineering strain rates $\left(0.005-20 \% . \mathrm{s}^{-1}\right)$. To explore the influence of the temperature, similar tensile tests were performed at various temperatures in the nematic domain $\left(20-80^{\circ} \mathrm{C}\right)$, at an engineering strain rate of 1 or $0.05 \% . \mathrm{s}^{-1}$, and with a maximum strain of $75 \%$.

As a result of the relatively low stiffness of the specimens, a high-frequency experimental noise of constant amplitude was observed. All the stressstrain curves were filtered using a Fast-Fourier Transform in Matlab (MathWorks, Natick, MA, USA) applying a low-pass filter with a cut-off frequency of $10 \mathrm{~Hz}$. In addition, all tensile curves at a 
strain rate higher than $1 \% \cdot \mathrm{s}^{-1}$ were averaged over at least three tests.

The tangent modulus $E_{t a n}$ was determined by the average slope of the tangents to the stress-strain curve for strains between 0 and $3 \%$.

\subsection{Analysis of the PM transition}

The change in slope due to the PM transition and the characteristics of the resulting transition region were identified by analyzing the uniaxial engineering stress-strain curves in Matlab. The general shape of the stress-strain curve changed significantly with strain rate and temperature. In addition, the end of the PM transition occurred gradually over a range of strains up to $3 \%$. The following method was used to robustly determine the limits of the transition region. A line from the middle strain to the beginning (or end) of the transition region was fitted on the curve for increasing strain ranges. This was repeated until the slope of this fitted line increased by $5 \%$ of its initial value. At this threshold, the line was deemed to leave the transition region and its tip marked the beginning (or end) of the transition. Finally, we defined the slope of the PM transition as the slope of the curve at the middle strain.

\subsection{Time-temperature superposition}

The viscoelasticity of thermorheologically simple materials obeys the empirical principle of timetemperature superposition (TTS) [24]. The TTS principle stems from the idea that thermorheologically simple materials present identical viscoelastic behaviors at equivalent pairs of time and temperature values. Master curves using the TTS principle have been built for the storage and loss moduli of LCEs in the nematic domain [1, 25, 26]. Similarly, we built the storage modulus master curves by shifting along the frequency axis each frequency sweep curve at a specific temperature in order to superimpose it with the neighboring temperatures curves (equ. (1)).

$$
\log \left(f_{T_{r e f}}\right)=\log \left(f_{T}\right)+\log \left(a_{r e f}(T)\right)
$$

where $T$ is the temperature, $f_{T_{\text {ref }}}$ and $f_{T}$ are the frequencies at the reference and actual temperatures, respectively, and $a_{r e f}(T)$ is the temperaturedependent shift factor of the material for the reference temperature $T_{\text {ref }}$.
The master curves were constructed for the nematic state only, as the time-temperature superposition does not generally apply through the nematic-isotropic transition [1].

Recently, Diani et al. [27] showed that the TTS principle extends to the strain rate dependence of the behavior of an amorphous polymer acrylate network, using shift factors determined from the frequency dependence. For the uniaxial tensile tests at multiple temperatures, the equivalent strain rate can be computed as,

$$
\dot{\varepsilon}\left(T_{\text {ref }}\right)=\frac{\dot{\varepsilon}(T)}{a_{\text {ref }}(T)},
$$

where $\dot{\varepsilon}$ is the engineering strain rate.

\section{Results and Discussion}

\subsection{TTS in frequency and time domain}

The master curves for the storage modulus and the loss factor $\tan \delta$, as well as the associated shift factor (Fig. 2) were determined from dynamic mechanical frequency sweep tests as described in sections 2.2 and 2.5. The shift factor was determined for the storage modulus and used to build the loss factor master curve.

Diani et al. [27] showed that, for a thermorheologically simple material, the TTS principle is verified in the rate domain as well as in the frequency domain using identical shift factors. Considering this hypothesis for the LCE studied, we evaluated the possible extension of the small strain characterization of the LCE to its large strain behavior. As a first validation, the curves for similar equivalent strain rates were compared (Fig. 3). Up to the PM transition, the curves superposed, which confirmed that the TTS principle can be broadly applied for all regions of the stress strain curves.

We next constructed a master curve of the tangent modulus $E_{t a n}$ for a reference temperature of $20^{\circ} \mathrm{C}$ (Fig. 4). The values of $E_{\text {tan }}$ at various temperatures and strain rates spanning the nematic domain were shifted according to the shift factors determined for the frequency dependence of the storage modulus. The $E_{\text {tan }}$ values superimposed well onto a common master curve, which increased slowly at small strain rates, before showing a large increase for strain rates above $0.1 \% . s^{-1}$. This master curve of $E_{\text {tan }}$ at $20^{\circ} \mathrm{C}\left(T / T_{n i} \approx 0.2\right)$ was qualitatively similar to the curve of the effective modulus 

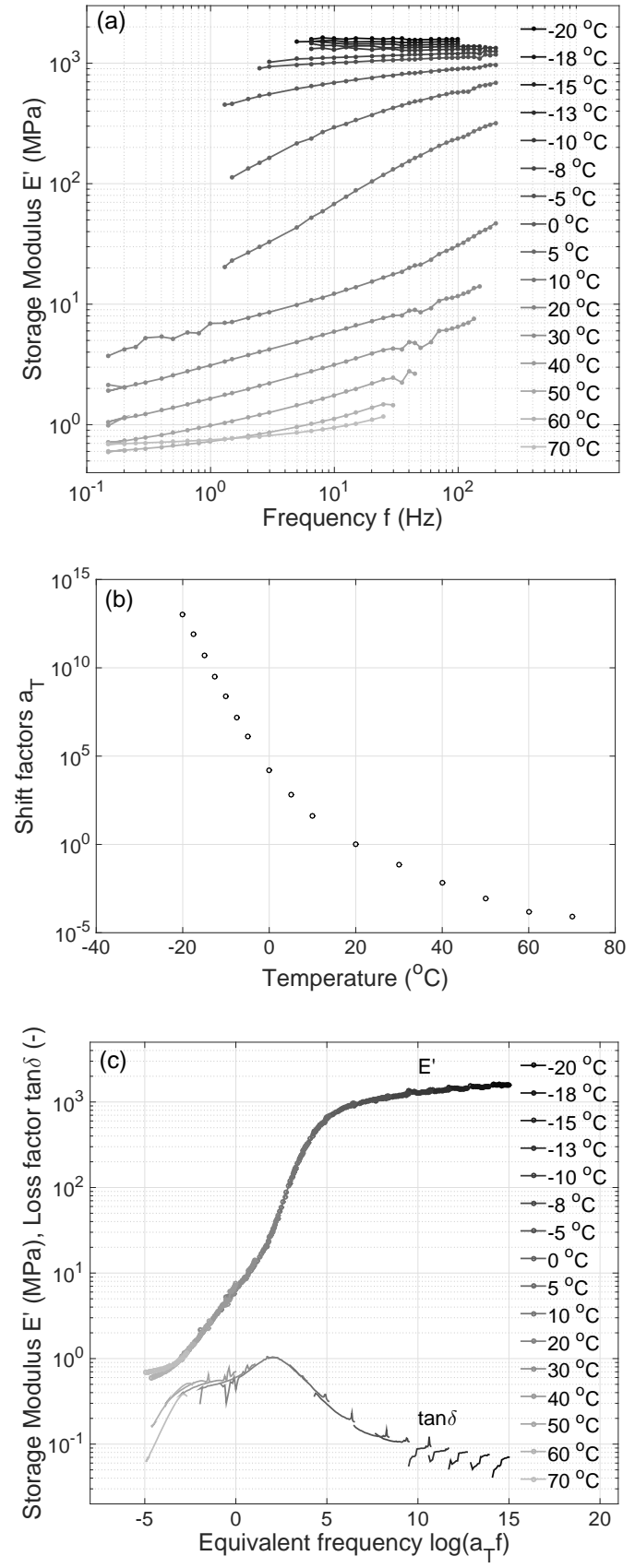

Figure 2: Dynamic mechanical behavior of the polydomainLCE measured at $0.1 \%$ strain amplitude: (a) storage modulus $E^{\prime}$ at different temperatures, (b) shift factor corresponding to the master curve of the storage modulus, and (c) master curves of the storage modulus $E^{\prime}$ and loss factor $\tan \delta$ obtained using the time-temperature superposition, $T_{\text {ref }}=20^{\circ} \mathrm{C}$.

versus strain rate at $69.8^{\circ} \mathrm{C}\left(T / T_{n i}=0.94\right)$ presented by Hotta and Terentjev [10]. Before the PM transition onset and under loading conditions close

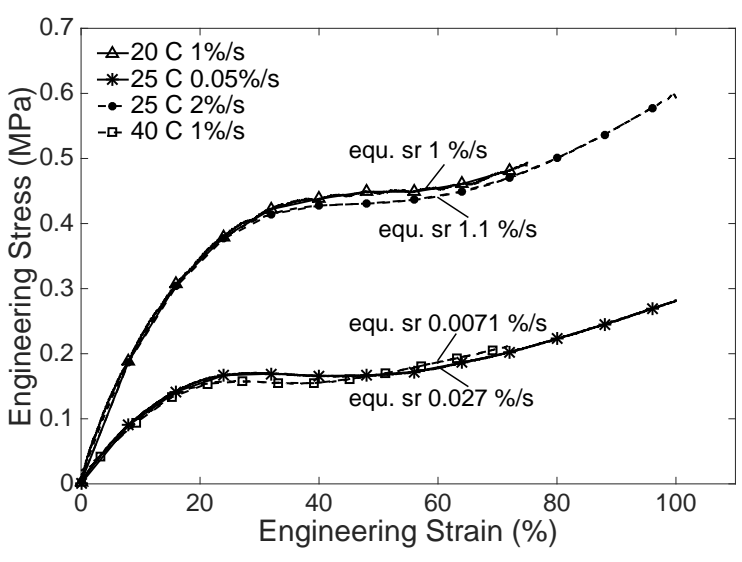

Figure 3: Stress-strain curves at equivalent strain rates, $T_{\text {ref }}=20^{\circ} \mathrm{C}$. The equ. $\mathrm{sr}$ value indicates the equivalent strain rate of each curve.

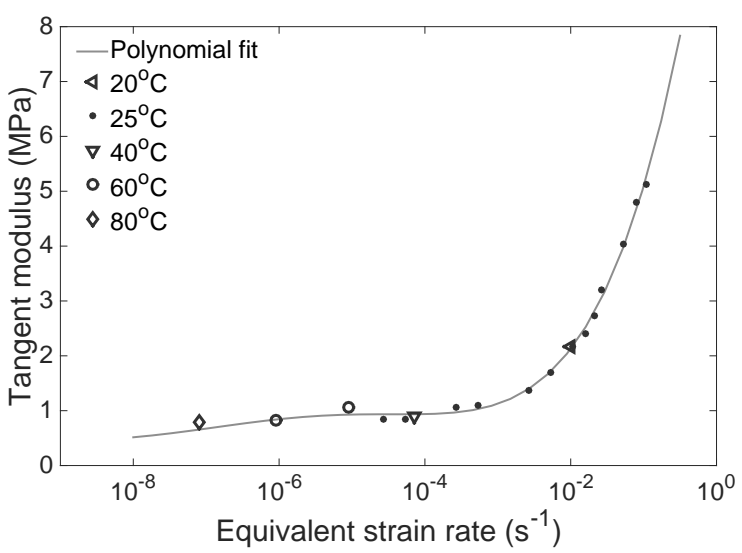

Figure 4: Master curve of the tangent modulus $E_{\text {tan }}, T_{\text {ref }}=$ $20^{\circ} \mathrm{C}$. The polynomial fit is a guide for the eye.

to equilibrium, LCEs behave like isotropic rubbers and their mechanical behavior has been described as isotropic Neo-Hookean [8]. The significant $E_{t a n}$ increase at higher strain rates was previously attributed to non-equilibrium nematic effects [10].

\subsection{Viscoelasticity of the PM transition}

The tensile behavior of the polydomain LCE at various strain rates showed the typical characteristics of semi-soft elasticity (Fig. 5). Each stressstrain curve exhibited a change in slope between 20 and $60 \%$ engineering strain corresponding to the PM transition. A change of the aspect of the specimen from opaque to transparent was likewise observed during the test.

Figure 6 shows the uniaxial mechanical behavior of the polydomain $\mathrm{LCE}$ at multiple temperatures in the nematic domain. Between $20^{\circ} \mathrm{C}$ and $60^{\circ} \mathrm{C}$, 


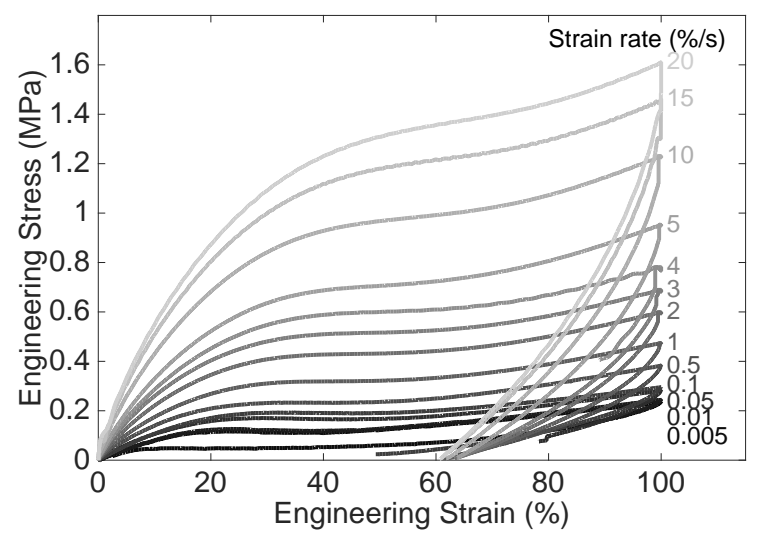

Figure 5: Uniaxial tensile behavior according to strain rate at room temperature.

the stress-strain curves exhibited a finite transition region. At $80^{\circ} \mathrm{C}$, the material was close to the nematic-isotropic transition, and although a PM transition was observed, no transition region was detected on the stress-strain curve.

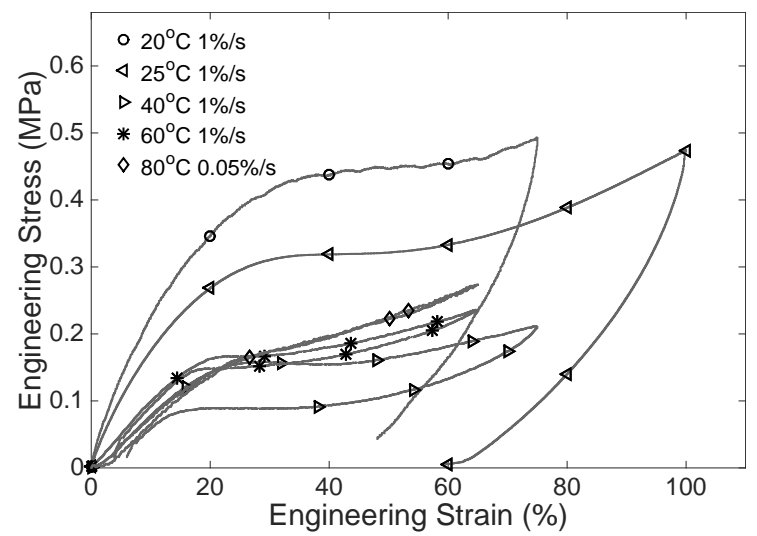

Figure 6: Uniaxial tensile behavior according to temperature.

We next constructed the master curves for the strain and stress levels at the onset and end of the transition as well as for the slope of the transition in figures 7,8 , and 9 , respectively.

The macroscopic mechanical state at the onset of, during, or at the end of the transition, consistently obeyed the TTS principle. This was also evidenced by the results in figure 3 , which showed that the stress-strain curves at equivalent strain rates were nearly identical across the PM transition. The TTS principle applies to this LCE independently of the transition and the state of the material, i.e. polydomain or monodomain. A similar observation has

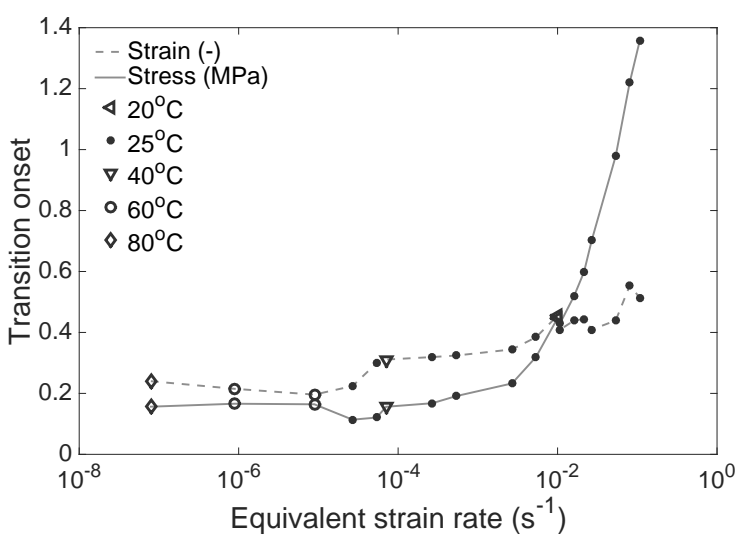

Figure 7: Master curve of the stress and strain at the onset of the transition, $T_{\text {ref }}=20^{\circ} \mathrm{C}$.

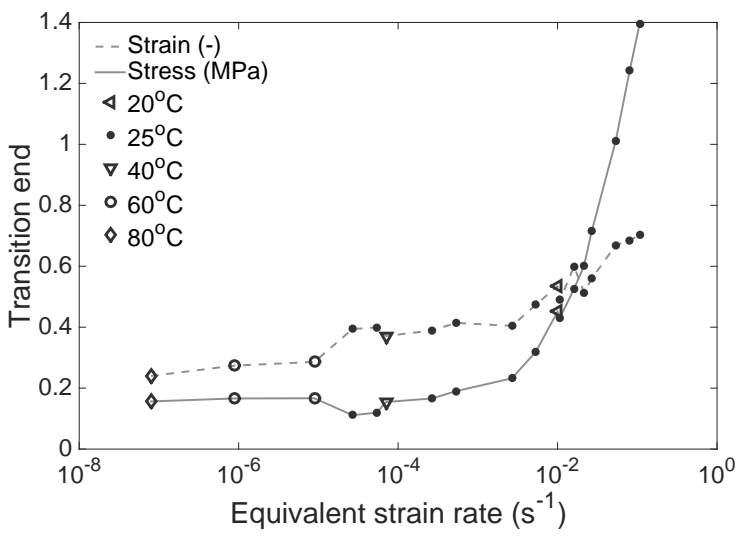

Figure 8: Master curve of the stress and strain at the end of the transition, $T_{r e f}=20^{\circ} \mathrm{C}$.

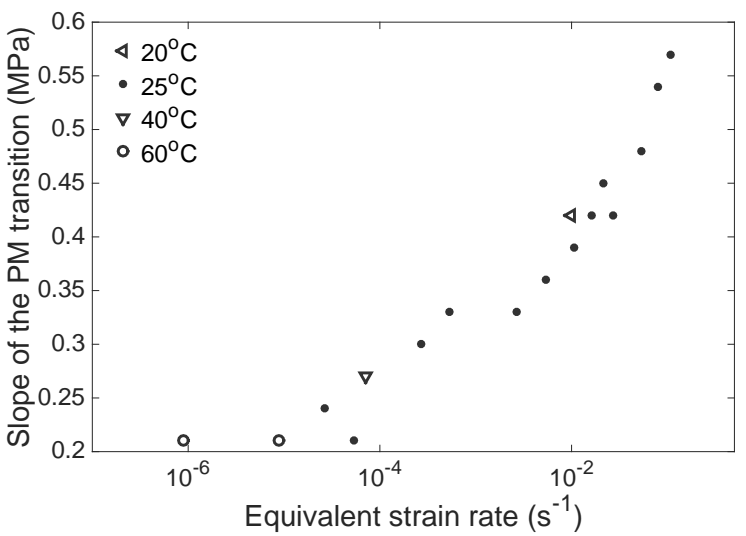

Figure 9: Master curve of the slope of the PM transition, $T_{\text {ref }}=20^{\circ} \mathrm{C}$

been made from the stress relaxation behavior of a stretched polydomain LCE [10].

The stress and strain at the end of the transition 
decreased with increasing temperature. This behavior has been previously measured and attributed to the decrease in chain anisotropy with increasing temperature [8]. In this study, the applicability of the TTS principle indicated an identical effect when decreasing the strain rate. Consequently, time-dependent phenomena control the end of the transition behavior.

Figure 10 shows the strain range of the PM transition region. Determining the onset and end of the transition is prone to errors (section 2.4). These errors are added in the computation of the length and only a trend can be obtained.

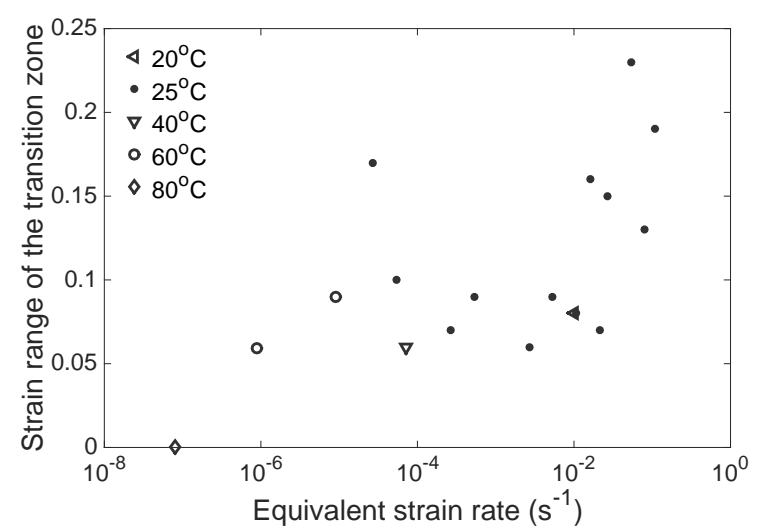

Figure 10: Master curve of the strain range of the transition at $T_{\text {ref }}=20^{\circ} \mathrm{C}$.

Similarly to previous studies [10-13], this trend, when extrapolated to equilibrium, i.e. infinitely small strain rates, shows that no transition region could be detected and the PM transition did not exhibit strain-softening but rather continued strain hardening. Away from equilibrium, the strain range of the PM transition tended to increase with the strain rate. In addition, a threefold increase of the slope of the PM transition was observed in this range of strain rates. This indicated that the soft elasticity, marked by an initial strain softening period, also partially disappeared at high strain rates (see also Fig. 5). The findings suggest that a significant chain orientation may develop along with mesogen orientation during the PM transition. The finite strain rate range of the transition region supports the conclusion that the soft elasticity of the PM transition, originating from the coupling between mesogens and polymer chains, belongs to a transient contribution to the mechanical behavior, in accordance with the suggestion made by Hotta and Terentjev [10].

\subsection{Strain recovery and relaxation mechanisms}

The strain recovery of the LCE was sensitive to temperature (Fig. 6). Below $40^{\circ} \mathrm{C}$, the PM transition was reversible during unloading, but the material required time at zero load to fully recover the applied deformation. For temperatures higher than $40^{\circ} \mathrm{C}$, the $\mathrm{PM}$ transition was reversible and nearly elastic. The hysteresis of the stress-strain curve decreased with increasing temperature, becoming nearly zero at $80^{\circ} \mathrm{C}$.

We next studied the dependence of the unloading and recovery behavior on strain rate at room temperature (Fig. 11). The unloading strain was measured as the unrecovered strain as the LCE specimen was unloaded to a zero stress; however, this strain was temporary, as the specimen would fully recover within 2 to 15 minutes if left unloaded. Both the recovery time and the unloading strain increased as expected with the strain rate up to $0.1 \% . s^{-1}$. Then, for strain rates higher than $0.5 \% . s^{-1}$, the unloading strain remained constant and independent of strain rate. The recovery time decreased with increasing strain rates above $0.1 \% . s^{-1}$.

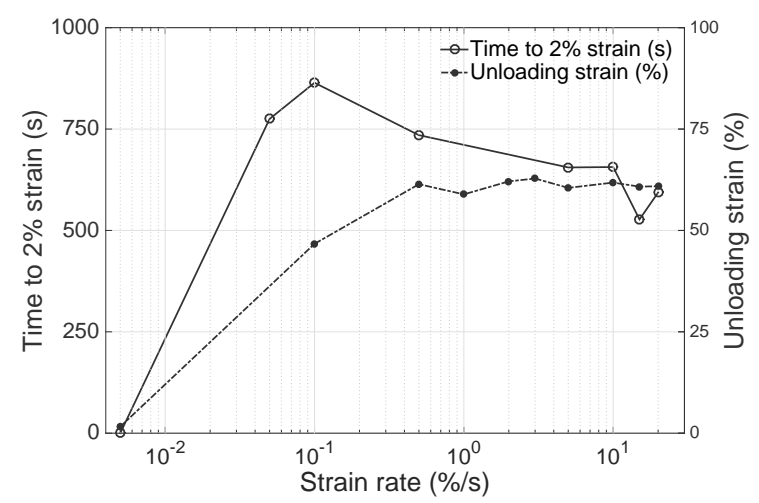

Figure 11: Unloading strain and recovery time after the PM transition.

The recovery behavior exhibited a classic influence of temperature on a viscoelastic material. As the relaxation times decrease with increasing temperature, a nearly elastic behavior was measured at high temperature or low strain rate (Figs. 6 and 11). However, the viscoelasticity of the elastomeric network alone cannot explain the saturation of the unloading strain for almost two decades of increasing strain rates. Hotta and Terentjev [10] arrived at a similar conclusion for the long time stress relaxation response. In addition, this phenomenon occurs over a longer time scale than previously mea- 
sured for the kinetics of mesogen rotation. Previous experiments for nematic side-chain LCEs suggest that the relaxation time for the mesogen reorientation is significantly smaller than the one measured here for the recovery time [20]. No direct measurement of the relaxation time associated with mesogen rotation has been performed in a main-chain nematic LCE. We expect the rotation of mesogens in main-chain LCEs to be slower than in side-chain LCEs, because of their incoroporation to the polymer backbone. However, the recovery time measured here was several orders of magnitude higher than the values associated with mesogen rotation. The saturation in the elastic recovery strain suggests additional time-dependent mechanisms with longer time scales. One of these mechanisms could be the slow relaxation associated with polydomain texture described in $[18,19]$.

Finally, Higaki et al. showed that a monodomain created by stretching a polydomain LCE with nematic genesis retains the subdomain texture [9]. Therefore, the texture of oriented monodomain LCEs could depend on the strain rate of the loading. The observed decrease in recovery time with increasing strain rate (Fig. 11) could then reflect a difference in texture. However, the effect of strain rate on the recovery behavior requires further investigation.

\section{Conclusion}

A main-chain LCE was synthesized and then cross-linked in the nematic polydomain state. Stretching the polydomain sample led to a transition to the monodomain state. The PM transition caused a soft elastic behavior, which was directly observed under uniaxial tension as a large increase in strain with small changes in stress. For most strain rate and temperature conditions, the soft region had an initial slight strain softening behavior.

The main finding of this work was that we were able to apply the temperature-dependent shift factor determined for small strain and in the frequency domain to create master curves for the large strainstress response in the strain rate domain. We were able to use the same shift factor to create master curves for the frequency-dependent storage and loss moduli, the rate-dependent effective modulus, the stress and strain levels at the onset and end of the soft elasticity region, and the length of the soft stress region.
For the main-chain polydomain LCE with nematic genesis, the soft elasticity of the PM transition is a transient phenomenon. This finding is consistent with those of Urayama et al. for nematic side-chain LCEs [19]. Additionally, the results suggest that the same mechanisms govern the timedependent behavior of the main-chain LCEs in the nematic phase over a wide range of strains, from well before and well after the soft elasticity region. This further suggests that the relaxation mechanisms of the network, and not of the mesogen orientation, dominate the rate-dependent behavior for the range of strain rates and temperatures examined here. However, the study of the unloading strain and recovery time highlighted the contribution of a relatively slow relaxation mode, suggested to be the relaxation of the polydomain texture.

\section{Acknowledgements}

TDN would like to acknowledge the funding support from the National Science Foundation (CMMI1130358). CY and MS also thank the National Science Foundation (CMMI-1350436). AA would like to thank the Postdoc Development Award 2014 of the Hopkins Extreme Materials Institute.

[1] M. Warner and E.M. Terentjev. Liquid Crystal Elastomers. Clarendon Press, Oxford, revised edition, 2007.

[2] H. Finkelmann, H.J. Kock, and G. Rehage. Investigations on liquid crystalline polysiloxanes 3 . Liquid crystalline elastomers - a new type of liquid crystalline material. Die Makromolekulare Chemie, Rapid Communications, 2(4):317-322, 1981.

[3] R. Zentel. Liquid crystalline elastomers. Advanced Materials, 1(10):321-329, 1989.

[4] M. Warner and X.J. Wang. Elasticity and phase behavior of nematic elastomers. Macromolecules, 24(17): 4932-4941, 1991.

[5] K. Hammerschmidt and H. Finkelmann. Stress-optical and thermomechanical measurements on liquid crystalline elastomers. Makromol. Chem., 190:1089-1101, 1989.

[6] J. Küpfer and H. Finkelmann. Nematic liquid single crystal elastomers. Die Makromolekulare Chemie, Rapid Communications, 12(12):717-726, 1991.

[7] S.M. Clarke, E.M. Terentjev, I. Kundler, and H. Finkelmann. Texture evolution during the polydomainmonodomain transition in nematic elastomers. Macromolecules, 31(15):4862-4872, 1998.

[8] S.V. Fridrikh and E.M. Terentjev. Polydomainmonodomain transition in nematic elastomers. Phys. Rev. E, 60(22):1847-1857, 1999.

[9] H. Higaki, K. Urayama, and T. Takigawa. Memory and Development of Textures of Polydomain Nematic Elastomers. Macromolecular Chemistry and Physics, 213(18):1907-1912, 2012. 
[10] A. Hotta and E.M. Terentjev. Long-time stress relaxation in polyacrylate nematic liquid crystalline elastomers. Journal of Physics: Condensed Matter, 13(50): 11453-11464, 2001.

[11] K. Urayama, E. Kohmon, M. Kojima, and T. Takigawa. PolydomainâLŚ Monodomain Transition of Randomly Disordered Nematic Elastomers with Different CrossLinking Histories. Macromolecules, 42(12):4084-4089, 2009.

[12] J.S. Biggins, M. Warner, and K. Bhattacharya. Elasticity of polydomain liquid crystal elastomers. Journal of the Mechanics and Physics of Solids, 60(4):573-590, 2012 .

[13] J.K. Whitmer, T.F. Roberts, R. Shekhar, N.L. Abbott, and J.J. de Pablo. Modeling the polydomainmonodomain transition of liquid crystal elastomers. Phys. Rev. E, 87(2):020502, 2013.

[14] J. Schätzle and W. Kaufhold. Nematic elastomers: The influence of external mechanical stress on the liquid-crystalline phase behavior. Die Makromolekulare Chemie, 190:3269-3284, 1989.

[15] J. Küpfer and H. Finkelmann. Liquid crystal elastomers: influence of the orientational distribution of the crosslinks on the phase behaviour and reorientation processes. Macromolecular Chemistry and Physics, 195 (4):1353-1367, 1994.

[16] C. Ortiz, C.K. Ober, and E.J. Kramer. Stress relaxation of a main-chain, smectic, polydomain liquid crystalline elastomer. Polymer, 39(16):3713-3718, 1998.

[17] S. Krause, F. Zander, G. Bergmann, H. Brandt, H. Wertmer, and H. Finkelmann. Nematic mainchain elastomers: Coupling and orientational behavior. Comptes Rendus Chimie, 12(1):85-104, 2009.

[18] S.M. Clarke and E.M. Terentjev. Slow stress relaxation in liquid crystal elastomers and gels. Faraday Discussions, 112:325-333, 1999.

[19] K. Urayama, S. Honda, and T. Takigawa. Slow dynamics of shape recovery of disordered nematic elastomers. Phys. Rev. E, 74(4):041709, 2006.

[20] M. Schonstein, W. Stille, and G. Strobl. Effect of the network on the director fluctuations in a nematic sidegroup elastomer analysed by static and dynamic light scattering. The European Physical Journal E - Soft Matter, 5:511-517, 2001.

[21] Dena M Agra-Kooijman, Michael R Fisch, Leela Joshi, Wanting Ren, Philip J McMullan, Anselm C Griffin, and Satyendra Kumar. Dual relaxation and structural changes under uniaxial strain in main-chain smectic-C liquid crystal elastomer. Physical Chemistry Chemical Physics, 17:191-199, 2015.

[22] C.M. Yakacki, M. Saed, D.P. Nair, T. Gong, and S.M. Reed. Tailorable and programmable liquid-crystalline elastomers using a two-stage thiol-acrylate reaction. RSC Advances, 5:18997-19001, 2015.

[23] M.O. Saed, A. H Torbati, D.P. Nair, and C.M. Yakacki. Synthesis of Programmable Main-chain Liquid-crystalline Elastomers Using a Two-stage Thiolacrylate Reaction. JoVE, 107:1-10, 2016.

[24] J.D. Ferry. Viscoelastic Properties of Polymers. Wiley, 1980.

[25] J.L. Gallani, L. Hilliou, and P. Martinoty. Mechanical behavior of side-chain liquid crystalline networks. Journal de Physique II, 6(3):443-452, 1996

[26] M. Giamberini, V. Ambrogi, P. Cerruti, and C. Carfagna. Viscoelasticity of main chain liquid crys- talline elastomers. Polymer, 47(13):4490-4496, 2006.

[27] J. Diani, P. Gilormini, and J.S. Arrieta. Direct experimental evidence of time-temperature superposition at finite strain for an amorphous polymer network. Polymer, 58:107-112, 2015. 
